\title{
Early Eocene marine ostracods from the Crescent Formation, southwestern Washington State, USA
}

\author{
TATSUHIKO YAMAGUCHI ${ }^{1, *} \&$ JAMES L. GOEDERT ${ }^{2}$ \\ ${ }^{1}$ Geosciences Research Division, Scripps Institution of Oceanography, University of California, San Diego, La Jolla, California 92093-0244, USA \\ ${ }^{2}$ Burke Museum of Natural History and Culture, University of Washington, Seattle, Washington 98195, USA \\ *Corresponding author (e-mail: tyamaguchi@mail.ucsd.edu/tyamaguch@mac.com)
}

\begin{abstract}
An assemblage of eleven species of Early Eocene marine intertidal and sublittoral ostracods has been found in the Crescent Formation in the Black Hills, Thurston County, Washington State, USA. The ostracods are subtropical and tropical taxa, such as Bairdoppilata, Cytherella and Neomonoceratina, which are found south of Washington today. Based on the geological age of the formation, the fauna corresponded to the Early Eocene Climatic Optimum. Five new species are recognized: Acanthocythereis olympiana sp. nov., Ambostracon irizukii sp. nov., Bairdoppilata crescentana sp. nov., Coquimba washingtonensis sp. nov. and Cytherelloidea squiresi sp. nov. This new assemblage from the Crescent Formation is the northernmost record of Early Eocene ostracods for the entire Pacific Basin. J. Micropalaeontol. 28(2): 117-129, November 2009.
\end{abstract}

KEYWORDS: climatic optimum, Crescent Formation, Early Eocene, Washington, Ostracoda

\section{INTRODUCTION}

The Early Eocene Climatic Optimum at c. 52 Ma was the warmest of the Cenozoic global climates. Oceanic water temperatures during that time were $c .8-19^{\circ} \mathrm{C}$ higher than they are today (Lear et al., 2000; Zachos et al., 2001; Lear, 2007) and had a profound effect on the distribution of shallow-marine biota and forced changes in faunal assemblage composition. For example, warm-water ostracod taxa that do not dwell in the Arctic Ocean were living there during the Early Pliocene Climatic Optimum (Cronin, 1991).

There are very few reports on Early Eocene ostracods from the North Pacific Basin (e.g. Carreño \& Cronin, 1993) and those from shallow-marine strata are from outcrops in California (Marianos \& Valentine, 1958) and drill cores from off the East China Sea (Liu, 1989; Yang et al., 1990). Herein we provide the first report on shallow-water ostracods from Early Eocene marine strata of the Crescent Formation in Washington State, USA and their taxonomic characters, and data on correlating sea temperatures and water depths are discussed. Much of the Crescent Formation was deposited during the Early Eocene Climatic Optimum and the fossils reported herein are the northernmost Early Eocene ostracods known from the entire North Pacific Basin (Fig. 1).

\section{STRATIGRAPHY AND GEOLOGICAL AGE}

The Crescent Formation is widely distributed in western Washington State and was named for outcrops at Crescent Bay on the north side of the Olympic Peninsula (Arnold, 1906). The Crescent Formation consists mainly of submarine basalt flows in the lower part, subaerial flows in the uppermost part, and is estimated to be over $15 \mathrm{~km}$ in thickness (Babcock et al., 1994). There are in some areas also thin interbeds of basaltic siltstone and sandstone that locally contain molluscan fossils (Squires \& Goedert, 1994).

The geological age of the Crescent Formation was determined using micro- and macrofossil data and radiometric methods. Armentrout \& Worsley (1980) examined calcareous nannofossils and assigned the upper part of the Crescent Formation to Zone NP16 (42.6-39.8 Ma; Luterbacher et al., 2004) of Martini (1971). Molluscan fossils of the lower Eocene informal 'Capay' Stage of Givens (1974) were reported (Squires \& Goedert 1994, 1996; Groves \& Squires, 1995) from the Crescent Formation in the Black Hills, western Washington State, from the same two sites (Fig. 1) that yielded the ostracodes studied herein. Squires (1988) correlated the 'Capay' Stage with planktonic foraminiferal Zones P7-8 (52.4-50.4 Ma; Luterbacher et al., 2004) of Berggren \& Miller (1988) and calcareous nannofossil Zones CP9-11 (54.8-49.0 Ma; Luterbacher et al., 2004) of Okada \& Bukry (1980). Squires \& Goedert (1996) also found benthic Foraminifera suggesting the Penutian Benthic Foraminiferal Stage of Mallory (1959). The Penutian Stage falls in the upper part of Zone CP9 to CP12 (Almgren et al., 1988). The basalt in the Crescent Formation within the Black Hills has been dated by the potassium-argon method as ranging in age from 51.4 to $55.6 \mathrm{Ma}$ using (Globerman et al., 1982). The radiometric data are consistent with the molluscan and benthic foraminiferal ages; however, the calcareous nannofossil biochronology of Armentrout \& Worsley (1980) is younger than ages indicated by the other methods. Armentrout \& Worsley (1980) reported the co-occurrence of Chiasmolithus solitus and Reticulofenestra bisecta, but, according to Berggren et al. (1995), these taxa do not occur together elsewhere in the world. The calcareous nannofossil data of Armentrout \& Worsley (1980) is thus in conflict with data from deep-sea records and needs to be re-examined. Here we regard the geological age of the Crescent Formation in the Black Hills as c. 52-50 Ma.

\section{MATERIAL AND METHODS}

Rock samples were collected from two separate localities in the Crescent Formation in the Black Hills, just south and west of Olympia, Washington (Fig. 1; Appendix A). The samples are muddy coarse-grained sandstone and sandy mudstone bearing abundant molluscan fossils. The institutional abbreviation used for localities is CSUN (California State University, Northridge). The CSUN collections have been moved to the Invertebrate Paleontology section, Natural History Museum of Los Angeles County, Los Angeles, California (LACMIP). CSUN loc. 1563 is registered with Burke Museum of Natural History and Culture, 

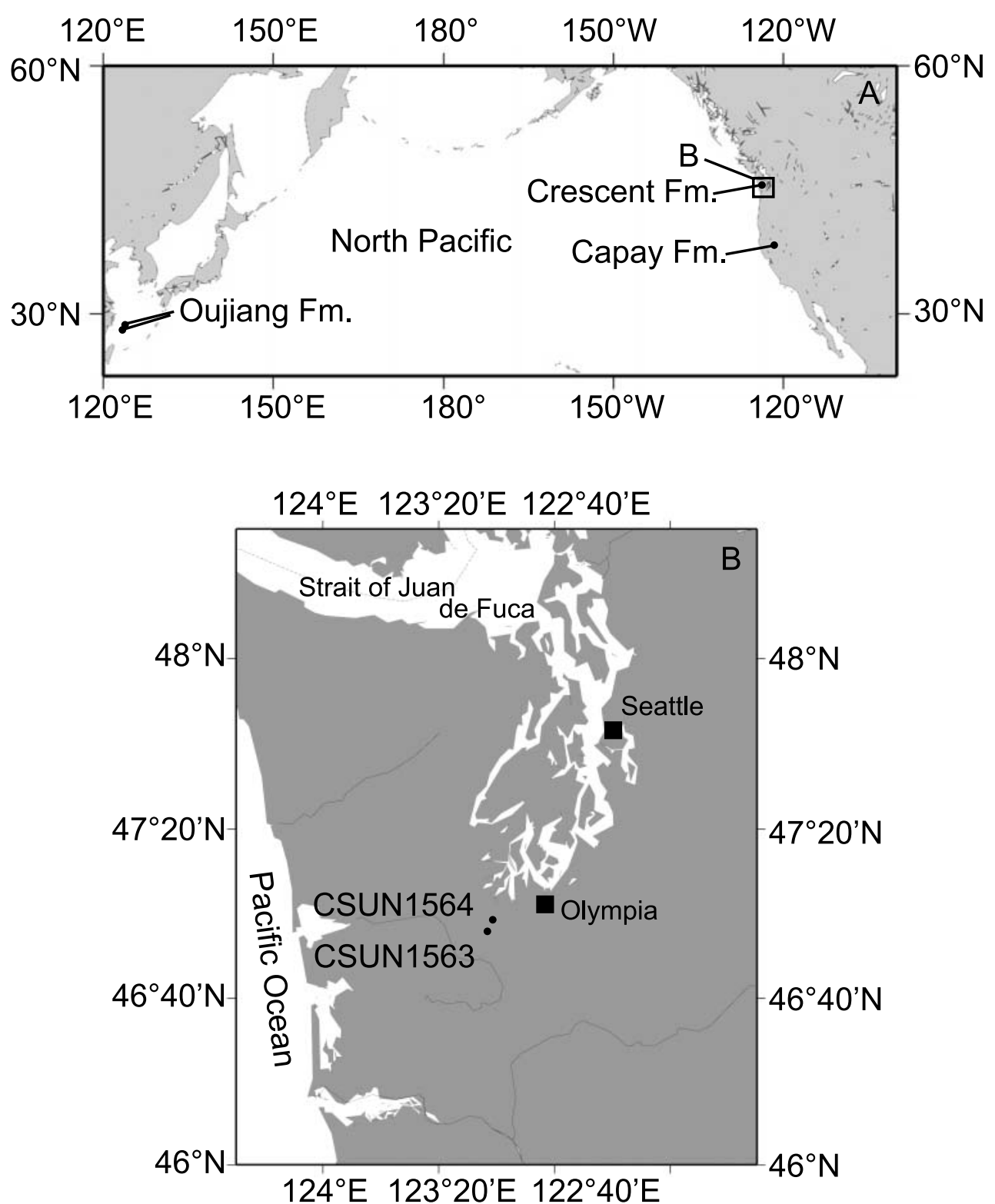

Fig. 1. Locations for Early Eocene ostracods in the North Pacific Ocean. (A) Locations and rock units for previous studies of Early Eocene ostracods as well as new study area in western North America. (B) Map showing localities in Washington State.

University of Washington, Seattle as locality B6169 'Larch Mountain'.

To extract ostracod specimens, $160 \mathrm{~g}$ and $444 \mathrm{~g}$ of rock samples were disaggregated using a saturated sodium sulphate solution and naphtha. The disaggregated samples were washed through a 250 mesh (63 $\mu$ m openings) sieve. Larger fractions of processed sample were dried with an isothermal chamber drier. Fossil ostracod specimens were picked from the fractions coarser than $125 \mu \mathrm{m}$. Ostracod species were identified with a binocular microscope at $70 \times$ magnification. Photographs of selected specimens were made with a scanning electron microscope, JEOL JSM-5310 at the Course of Earth Science, Kanazawa University.

To observe marginal pore-canals and hingements, photographs of specimens were captured with a transmitted micro- scope, a digital camera Olympus DP70, and software (Dynamic Eye Real of Mitani Co. Ltd) for focus image composition at the Course of Earth Science, Kanazawa University.

\section{FOSSIL OSTRACODS}

One hundred and five ostracod fossils (83 carapaces and 22 valves) were recovered from two samples. Many of the fossils are ochre and white in colour, whereas some are translucent and most specimens are well preserved. Eleven species were identified (Table 1). The sample from CSUN loc. 1563 yielded the largest number of specimens. The assemblage from CSUN loc. 1563 includes: Ambostracon irizukii sp. nov. (24\% of 93 specimens), Bairdoppilata crescentana sp. nov. (20\%), Neonesidea? sp. (22\%), Coquimba washingtonensis sp. nov. (11\%), Acanthocythereis olympiana sp. nov. (7\%), Cytherella sp., Cytherelloidea squiresi 


\begin{tabular}{|c|c|c|c|c|c|c|}
\hline \multirow{2}{*}{$\begin{array}{l}\text { Sample } \\
\text { Dry weight }(\mathrm{g}) \\
\text { Type of specimen }\end{array}$} & \multicolumn{3}{|c|}{$\begin{array}{c}\text { CSUN1563 } \\
444\end{array}$} & \multicolumn{3}{|c|}{$\begin{array}{c}\text { CSUN1564 } \\
160\end{array}$} \\
\hline & $\mathrm{C}$ & $\mathrm{L}$ & $\mathrm{R}$ & $\mathrm{C}$ & $\mathrm{L}$ & $\mathrm{R}$ \\
\hline \multicolumn{7}{|l|}{ Species } \\
\hline Acanthocythereis olympiana sp. nov. & 4 & & & & & \\
\hline Ambostracon irizukii sp. nov. & 16 & 1 & & 1 & & \\
\hline Bairdoppilata crescentana sp. nov. & 24 & 2 & 7 & 7 & 2 & 1 \\
\hline Coquimba washingtonensis sp. nov. & 11 & & 1 & 1 & & \\
\hline Cytherella sp. & & 3 & & & & \\
\hline Cytherelloidea squiresi sp. nov. & 4 & 2 & 1 & & & \\
\hline Neomonoceratina sp. & & & 1 & & & \\
\hline Neonesidea? sp. & 9 & 1 & & & & \\
\hline Paradoxostoma sp. & 1 & & & & & \\
\hline Parakrithella sp. & 2 & & & & & \\
\hline Xestoleberis sp. & 3 & & & & & \\
\hline
\end{tabular}

$\mathrm{C}$, carapace; $\mathrm{L}$, left valve; $\mathrm{R}$, right valve.

Table 1. List of ostracode species from the Crescent Formation, CSUN locs 1563 and 1564 .

sp. nov. and Neomonoceratina sp. are also included $(<5 \%)$. Species of Paradoxostoma, Xestoleberis and Parakrithella were also found, but specimens are few, and cannot be identified further.

\section{SYSTEMATIC DESCRIPTIONS OF SELECTED TAXA}

Type specimens are housed at the National Museum of Natural History, Smithsonian Institution, Washington, D.C. (USNM). Paratype specimens from this study are also deposited in the collections of the Burke Museum, University of Washington, Seattle (UWBM). Morphological terminology in the descriptions follows the scheme reviewed by Athersuch et al. (1989) and Horne et al. (2002). The classification of taxonomic ranks higher than the generic rank follows Hartmann \& Puri (1974). Specimens were measured with a micrometre ruler under a binocular microscope. Measurements of length and height are derived from both carapace and valve specimens, whereas those of width were obtained from carapace specimens. The division of size is as follows: small size means length less than $0.45 \mathrm{~mm}$; medium size indicates a length between $0.45 \mathrm{~mm}$ and $0.75 \mathrm{~mm}$; large indicates that length exceeds $0.75 \mathrm{~mm}$. Abbreviations used are $\mathrm{L}$, length; $\mathrm{H}$, height; and $\mathrm{W}$, width.

Order Platycopida Sars, 1866

Superfamily Cytherelloidea Sars, 1866

Family Cytherellidae Sars, 1866

Genus Cytherelloidea Alexander, 1929

Cytherelloidea squiresi sp. nov.

(Pl. 1, figs 1-6)

Derivation of name. In honour of Dr Richard L. Squires (California State University) for his studies of fossil molluscs from the Pacific Coast of North America.

Diagnosis. A Cytherelloidea ornamented with two horizontal carinae, a narrower anterior marginal rim and characterized by a flattened posterior end in the dorsal view.

Holotype. USNM 537683, adult carapace, from CSUN loc. 1563.
Paratypes. USNM 537684, adult right valve, from CSUN loc. 1563; USNM 537685, adult right valve, from CSUN loc. 1563; UWBM no. 98690, adult carapace, from CSUN loc. 1563.

Type locality and horizon. CSUN loc. 1563. See also Appendix A.

Description. Carapace slightly robust and medium sized. Lateral outline elliptical: anterior and posterior margins round; dorsal margin straight or slightly sinuous; ventral margin curved and concave near middle. Maximum length across middle. Maximum height across anterodorsal corner.

Surface ornamented with two horizontal carinae, irregular reticulation and narrow marginal rim. The carinae parallel each other and are curved convexly near middle. Upper carina across central area; lower carina on ventral area. Reticulation present between lower carina and ventral margin and formed by irregular fossae. Posterior marginal rim developed. Marginal denticles project from posterior margin.

Internally, sinuate hingement line. Hingement adont-type. In left valve, socket on median element.

Dorsal outline elongated and subtrapezoidal: anterior and posterior ends flattened; lateral margins curved and turn to straight near one-fifth from anterior end. Right valve dorsally overlapped by left.

Dimensions. USNM 537683, $\mathrm{L}=0.69 \mathrm{~mm}, \mathrm{H}=0.37 \mathrm{~mm}, \mathrm{~W}=$ $0.29 \mathrm{~mm}$; USNM 537684, L=0.65 mm, H=0.37 mm; USNM 537685, L=0.63 mm, H=0.35 mm; UWBM no. 98690, L= $0.65 \mathrm{~mm}, \mathrm{H}=0.37 \mathrm{~mm}, \mathrm{~W}=0.21 \mathrm{~mm}$.

Remarks. This new species resembles Cytherelloidea californica Le Roy, 1943 and C. directiangular Holden, 1964 in the lateral outline and two horizontal carinae. Cytherelloidea californica and $C$. directiangular were originally described from the Middle Pleistocene Lomita Marl Member of the San Pedro Formation and the Upper Cretaceous Rosario Formation in California, respectively. Cytherelloidea squiresi is smaller than either of the two species from California. Cytherelloidea squiresi is also distinguishable from $C$. directiangular by having undeveloped marginal rims and differs from $C$. californica in having narrower carinae in the lateral view, and a narrower and distinct anterior marginal rim and flattened posterior end in dorsal view.

Order Podocopida Sars, 1866

Suborder Bairdiocopina Sars, 1888

Superfamily Bairdoidea Sars, 1888

Family Bairdiidae Sars, 1888

Genus Bairdoppilata Coryell, Sample \& Jennings, 1935, emended Maddocks, 1969

Bairdoppilata crescentana sp. nov.

(P1. 1, figs 7-13)

Derivation of name. Named after the Crescent Formation.

Diagnosis. A Bairdoppilata characterized by slightly high carapace with round anterior margin and caudal process below middle of posterior margin and nine muscle-scars within left valve. 


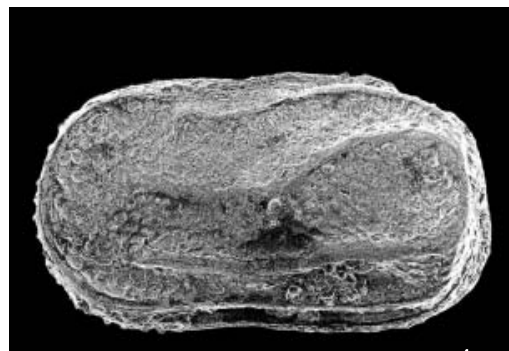

1
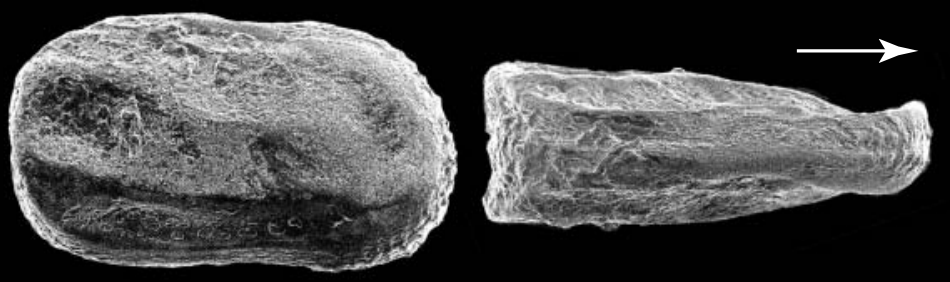

2

3
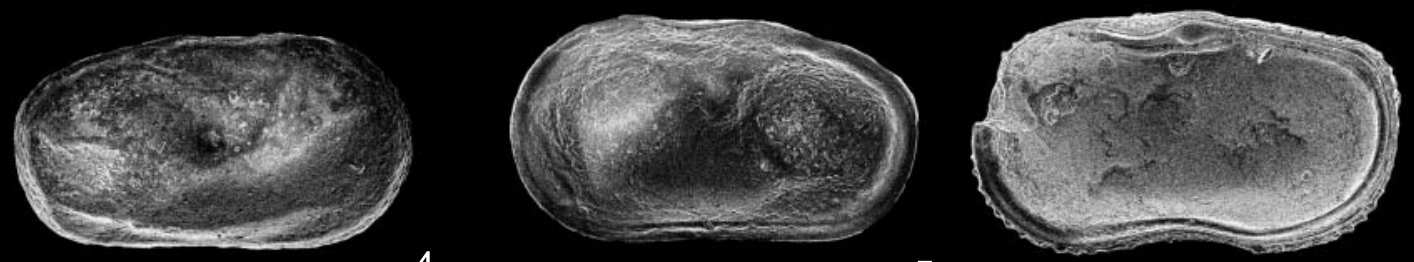

4

5

6
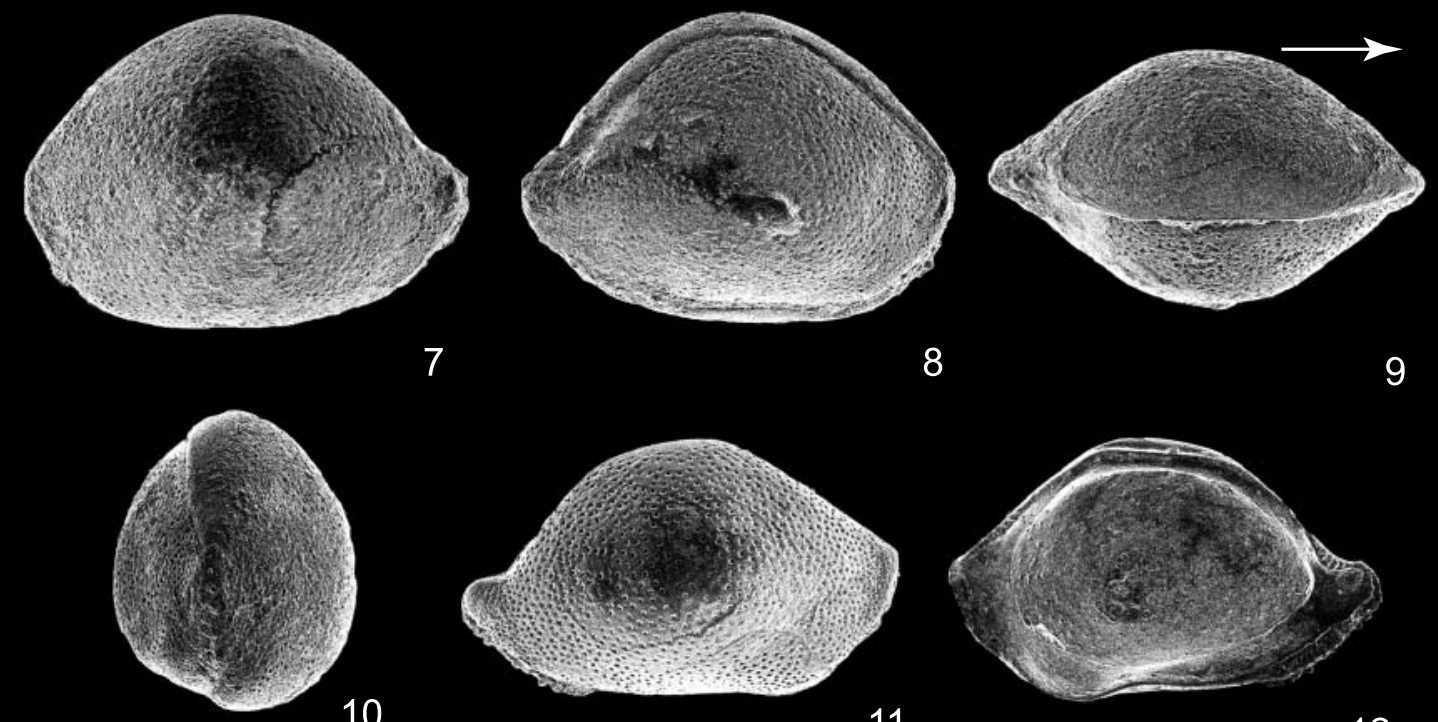

11

12
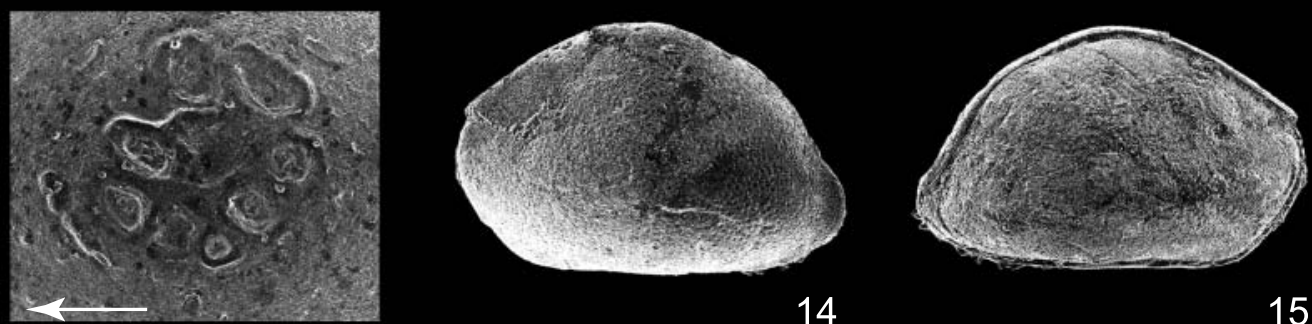

14

15

13

$7-12,14,15$

13

Explanation of Plate 1.

figs 1-6. Cytherelloidea squiresi sp. nov.: 1, left lateral view, holotype, USNM 537683, adult carapace; 2, right lateral view, holotype, USNM 537683, adult carapace; $\mathbf{3}$, dorsal lateral view, holotype, USNM 537683, adult carapace; $\mathbf{4}$, external view, paratype, USNM 537685, adult right valve; 5, internal view, paratype, USNM 537685, adult right valve; 6, internal view, paratype, USNM 537684, adult right valve. figs 7-13. Bairdoppilata crescentana sp. nov.: 7, left lateral view, holotype, USNM 537686, adult carapace; 8, right lateral view, holotype, USNM 537686, adult carapace; 9, dorsal view, holotype, USNM 537686, adult carapace; 10, anterior view, holotype, USNM 537686, adult carapace. 11, external view, paratype, USNM 537688, adult valve; 12, internal view, paratype, USNM 537688, adult valve; 13, muscle scars in adult right valve, paratype, USNM 537688. figs 14-15. Neonesidea? sp., USNM 537689, adult carapace: 14, left lateral view; 15, right lateral view. Scale bars $0.1 \mathrm{~mm}$. Arrows indicate anterior direction. All specimens are from CSUN loc. 1563. 


\begin{tabular}{|c|c|c|c|c|c|c|}
\hline Species & Ontogenic stage & & Number & Range (mm) & Average (mm) & Standard deviation \\
\hline \multirow[t]{4}{*}{ Cytherelloidea squiresi sp. nov. } & Adult & $\mathrm{L}$ & 4 & $0.63-0.69$ & 0.66 & 0.022 \\
\hline & & $\mathrm{H}$ & 4 & $0.35-0.37$ & 0.37 & 0.0087 \\
\hline & & $\mathrm{H} / \mathrm{L}$ & 4 & $0.54-0.57$ & 0.56 & 0.012 \\
\hline & & W/L & 2 & $0.32-0.42$ & 0.37 & 0.049 \\
\hline \multirow{2}{*}{ Bairdoppilata crescentana sp. nov. } & Adult & $\mathrm{L}$ & 20 & $0.92-1.15$ & 1.04 & 0.087 \\
\hline & & $\mathrm{W} / \mathrm{L}$ & 19 & $0.47-0.60$ & 0.56 & 0.048 \\
\hline \multirow[t]{5}{*}{ Neonesidea? sp. } & Adult & $\mathrm{L}$ & 6 & $0.86-1.01$ & 0.92 & 0.045 \\
\hline & & $\mathrm{H}$ & 6 & $0.52-0.61$ & 0.57 & 0.032 \\
\hline & & $\mathrm{W}$ & 5 & $0.53-0.61$ & 0.57 & 0.027 \\
\hline & & $\mathrm{H} / \mathrm{L}$ & 6 & $0.57-0.67$ & 0.61 & 0.043 \\
\hline & & $\mathrm{W} / \mathrm{L}$ & 5 & $0.58-0.71$ & 0.62 & 0.048 \\
\hline \multirow[t]{10}{*}{ Ambostracon irizukii sp. nov. } & Adult & $\mathrm{L}$ & 5 & $0.82-0.94$ & 0.90 & 0.060 \\
\hline & & $\mathrm{H}$ & 5 & $0.51-0.54$ & 0.52 & 0.026 \\
\hline & & $\mathrm{W}$ & 5 & $0.41-0.52$ & 0.49 & 0.059 \\
\hline & & $\mathrm{H} / \mathrm{L}$ & 5 & $0.56-0.63$ & 0.58 & 0.026 \\
\hline & & $\mathrm{W} / \mathrm{L}$ & 5 & $0.51-0.57$ & 0.55 & 0.030 \\
\hline & A-1 & $\mathrm{L}$ & 11 & $0.69-0.79$ & 0.67 & 0.043 \\
\hline & & $\mathrm{H}$ & 11 & $0.33-0.36$ & 0.34 & 0.012 \\
\hline & & $\mathrm{W}$ & 10 & $0.23-0.33$ & 0.27 & 0.025 \\
\hline & & $\mathrm{H} / \mathrm{L}$ & 11 & $0.48-0.58$ & 0.53 & 0.034 \\
\hline & & $\mathrm{W} / \mathrm{L}$ & 10 & $0.35-0.48$ & 0.37 & 0.12 \\
\hline \multirow[t]{3}{*}{ Coquimba washingtonensis sp. nov. } & Adult & $\mathrm{L}$ & 12 & $0.69-0.79$ & 0.73 & 0.033 \\
\hline & & $\mathrm{H}$ & 12 & $0.34-0.40$ & 0.37 & 0.018 \\
\hline & & W & 12 & $0.35-0.41$ & 0.38 & 0.017 \\
\hline
\end{tabular}

Table 2. Measurements of ostracode species from the Crescent Formation.

Holotype. USNM 537686, adult carapace from CSUN loc. 1563.

Paratypes. USNM 537687, adult carapace from CSUN loc. 1563; USNM 537688, adult right valve from CSUN loc. 1563; UWBM no. 98691, adult carapace from CSUN loc. 1563; UWBM no. 98692, adult right valve from CSUN loc. 1563.

Material. Eighteen adult carapaces, one adult left valve and one adult right valve, including types.

Type locality and horizon. CSUN loc. 1563. See also Appendix A.

Description. Carapace robust and large. In external view, lateral outline subpentagonal: anterior margin round; posterior margin tapering below middle of carapace, forming caudal process; dorsal margin arched; ventral margin straight. Maximum length across caudal process; maximum height across middle of carapace. Surface ornamented with punctae. Dorsal outline lenticu- lar: anterior and posterior ends tapering; lateral margins curved. Anterior outline elliptical: lateral margins curved symmetrically; dorsal and ventral margins curved with terraces at contact of left and right valves. Contact line between left and right valves sinuous.

In internal view of right valve, anterior and posteroventral marginal zones broad. Hingement lophodont-type: left valve's hingement composed of at least three teeth as anterior element, smooth bar as median element, and seven teeth as posterior element. Adductal muscle scars ovate. Eight scars arranged around one scar concentrically.

Dimensions. See also Table 2. USNM 537686, L=0.99 mm, $\mathrm{H}=0.70 \mathrm{~mm}, \mathrm{~W}=0.60 \mathrm{~mm}$; USNM 537687, L=0.93 $\mathrm{mm}, \mathrm{H}=$ $0.65 \mathrm{~mm}, \mathrm{~W}=0.54 \mathrm{~mm}$; USNM $537688, \mathrm{~L}=0.98 \mathrm{~mm}, \mathrm{H}=0.59 \mathrm{~mm}$; UWBM no. $98691, \mathrm{~L}=1.05 \mathrm{~mm}, \mathrm{H}=0.70 \mathrm{~mm}, \mathrm{~W}=0.52 \mathrm{~mm}$; UWBM no. $98692, \mathrm{~L}=0.92 \mathrm{~mm}, \mathrm{H}=0.55 \mathrm{~mm}$.

Occurrence. CSUN locs 1563 and 1564. 
Remarks. Bairdoppilata crescentana sp. nov. resembles B. cratericola Maddocks, 1969 and B. cretacea (van Veen, 1936) in lateral outline, especially outlines of the anterior and posterior margins. Bairdoppilata cratericola was originally described on the basis of living specimens from Madagascar and B. cretacea (van Veen, 1936) was found in European Cretaceous strata and the Upper Cretaceous Rosario Formation of California (Holden, 1964). Bairdoppilata crescentana can be distinguished from $B$. cratericola (size range of single valve: $\mathrm{L}=0.88-0.89 \mathrm{~mm}$, $\mathrm{H}=0.50-0.59 \mathrm{~mm}$; Maddocks, 1969) by having a larger carapace and one muscle-scar more than the modern species. Bairdoppilata crescentana differs from $B$. cretacea (size range of single valve: $\mathrm{L}=0.88-0.92 \mathrm{~mm}, \mathrm{H}=0.45-0.65 \mathrm{~mm}, \mathrm{~W}=0.21-0.27 \mathrm{~mm}$; Holden, 1964) in having a wider and slightly higher carapace and two muscle-scars less than the Cretaceous species.

$$
\begin{aligned}
& \text { Genus Neonesidea Maddocks, } 1969 \\
& \text { Neonesidea? sp. } \\
& \text { (P1. 1, figs 13-15; P1. 2, figs 1-3) }
\end{aligned}
$$

Material. Six adult carapaces including USNM 537689 and three A-1 carapaces containing USNM 537690 from CSUN loc. 1563.

Description. Carapace robust and large. Lateral outline subhexagonal: anterior margin round; posterior margin round, having apex below middle of this margin; dorsal margin gently arched; ventral margin straight or slightly curved. Maximum length across middle of carapace; maximum height across anterodorsal corner. Surface ornamented with punctae. Dorsal outline elliptical: anterior and posterior ends tapering; lateral margins curved. Anterior outline subovate: lateral margins curved asymmetrically; dorsal margin arched with terrace at contact of left and right valves; ventral margins sinuous and tapering at contact of two valves. Contact between valves sinuous.

Right valve dorsally overlapped by left valve. A-1 carapace lower $\mathrm{H} / \mathrm{L}$ and $\mathrm{W} / \mathrm{L}$ ratios than adult.

Dimensions. See also Table 2. USNM 537689, L=0.91 mm, $\mathrm{H}=0.57 \mathrm{~mm}, \mathrm{~W}=0.53 \mathrm{~mm}$; USNM 537690, $\mathrm{L}=0.85 \mathrm{~mm}$, $\mathrm{H}=0.45 \mathrm{~mm}, \mathrm{~W}=0.49 \mathrm{~mm}$.

Occurrence. CSUN loc. 1563.

Remarks. Due to uncertainty regarding interior characters of this species, the generic assessment is tentative. The elongated hexagonal lateral outline is characteristic of Neonesidea. The species resembles $N$. sitkagi Brouwers, 1990 and $N$. antonbruuna Maddocks, 1969 in lateral outline, described from modern specimens from Alaska and Madagascar, respectively. Neonesidea? sp. can be distinguished from $N$. antonbruuna by having larger carapace, but without more data this species from the Crescent Formation cannot be identified with certainty.

Superorder Cytherocopina Baird, 1850

Superfamily Cytheroidea Baird, 1850

Family Cytheridae Baird, 1850

Genus Neomonoceratina Kingma, 1948
Neomonoceratina $\mathrm{sp}$.

(P1. 2, figs 4, 5)

Material. USNM 537691, adult right valve from CSUN loc. 1563.

Description. Carapace slightly robust and medium in size. In external view, lateral outline ovate: anterior margin round; posterior margin round, having apex above middle of this margin; dorsal margin arched, having angular at anterodorsal corner; ventral margin curved.

Surface ornamented with reticulation and dorsomedian depression. Reticulation composed of secondary reticulation framed by distinct polygonal muri. Secondary reticulation formed by round fossae and faint muri. Secondary reticulation on anterior, posterior and ventral areas finer than central area. Dorsomedian depression divided into upper and lower parts by carina. This carina binding between muri of reticulation. Upper part of depression larger than lower. Posterior one-quarter of ventral area slightly projected resembling ala.

In internal view of right valve, schizodont-type hinge: anterior element composed of two-node tooth and socket; median element smooth; posterior element round socket. Anterior marginal zone broad.

Dimensions. USNM 537691, L=0.70 mm, H=0.47 mm.

Occurrence. CSUN loc. 1563.

Remarks. This species is similar to Neomonoceratina ikeyai Irizuki \& Yamada in Irizuki et al. (2004) in lateral outline. $N$. ikeyai was originally described from the lower Miocene Akeyo Formation in central Japan and differs from Neomonoceratina sp. by having a larger carapace and coarser reticulation. Until more specimens can be found, the species from the Crescent Formation cannot be identified more precisely.

Family Hemicytheridae Puri, 1953

Genus Ambostracon Hazel, 1962

Ambostracon irizukii sp. nov.

(Pl. 2, figs 6-13; Fig. 2)

Derivation of name. In honour of Dr Toshiaki Irizuki (Shimane University), who studies taxonomy and phylogeny of the family Hemicytheridae.

Diagnosis. An Ambostracon characterized by less reticulation on the anterior area and regular reticulation formed by polygonal fossae and narrower muri.

Holotype. USNM 537692, adult carapace from CSUN loc. 1563.

Paratypes. USNM 537693, adult carapace from CSUN loc. 1563; USNM 537694, A-1 carapace from CSUN loc. 1563; USNM 537695, A-1 carapace from CSUN loc. 1563; USNM 537696, A-1 left valve from CSUN loc. 1563; UWBM no. 98693 , adult carapace from CSUN loc. 1563; UWBM no. 98694, A-1 carapace from CSUN loc. 1563. 


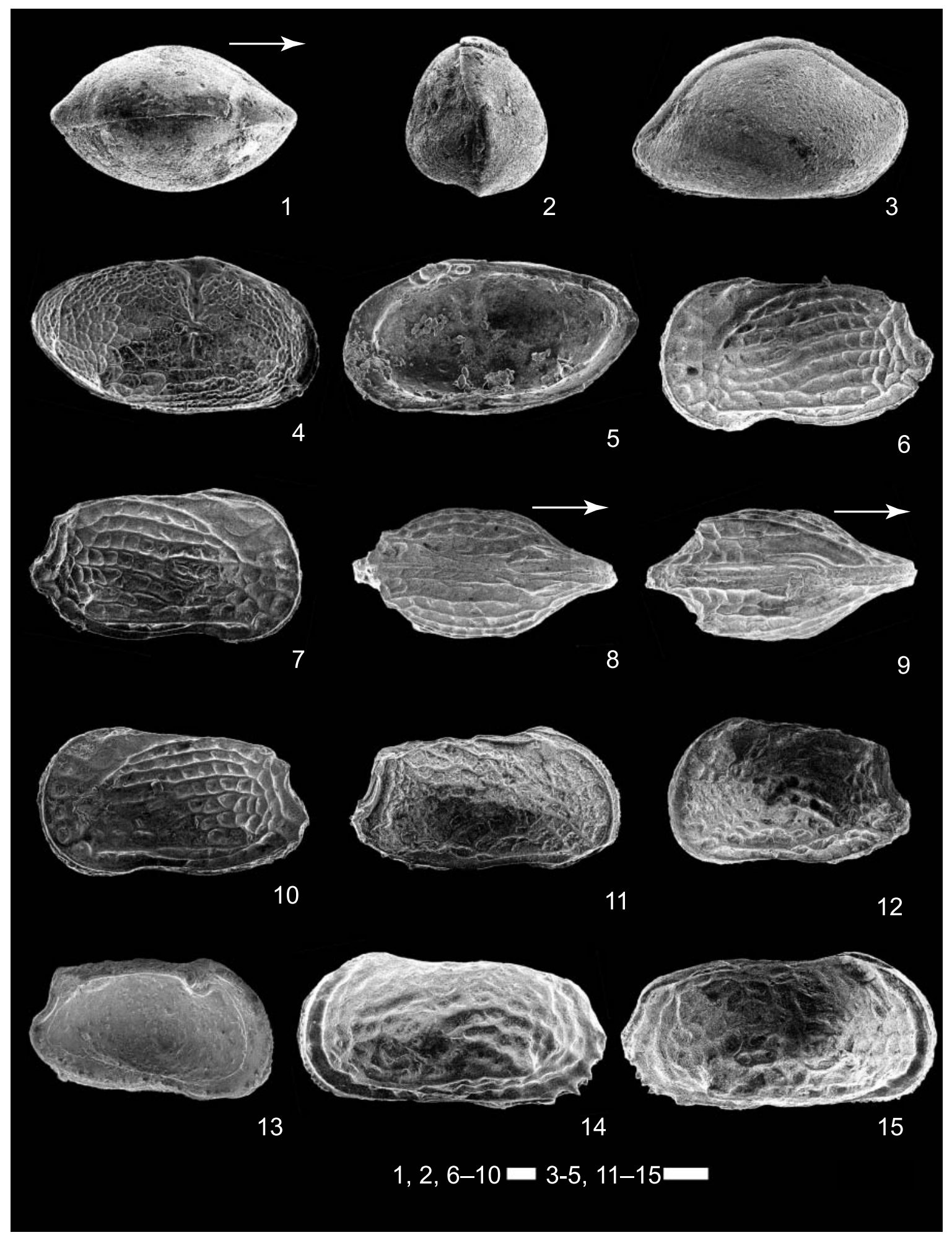

Explanation of Plate 2.

figs 1-3. Neonesidea? sp.: 1, dorsal view, USNM 537689, adult carapace, 2, anterior view, USNM 537689, adult carapace. 3, right lateral view, USNM 537690, A-1 carapace. figs 4, 5. Neomonoceratina sp., USNM 537691, adult right valve: 4, external view; 5, internal view. figs 6-13. Ambostracon irizukii sp. nov.: 6, left lateral view, holotype, USNM 537692, adult carapace; 7, right lateral view, holotype, USNM 537692, adult carapace; 8, dorsal view, holotype, USNM 537692, adult carapace; 9, ventral view, holotype, USNM 537692, adult carapace; 10, left lateral view, paratype, USNM 537693, adult carapace; 11, right lateral view, paratype, USNM 537694, A-1 carapace; 12, left lateral view, paratype, USNM 537695, A-1 carapace; 13, internal view, paratype, USNM 537696, A-1 left valve. figs 14, 15. Coquimba washingtonensis sp. nov., holotype, USNM 537697: 14, left lateral view; 15, right lateral view. Scale bars $0.1 \mathrm{~mm}$. Arrows indicate anterior direction. All specimens are from CSUN loc. 1563. 

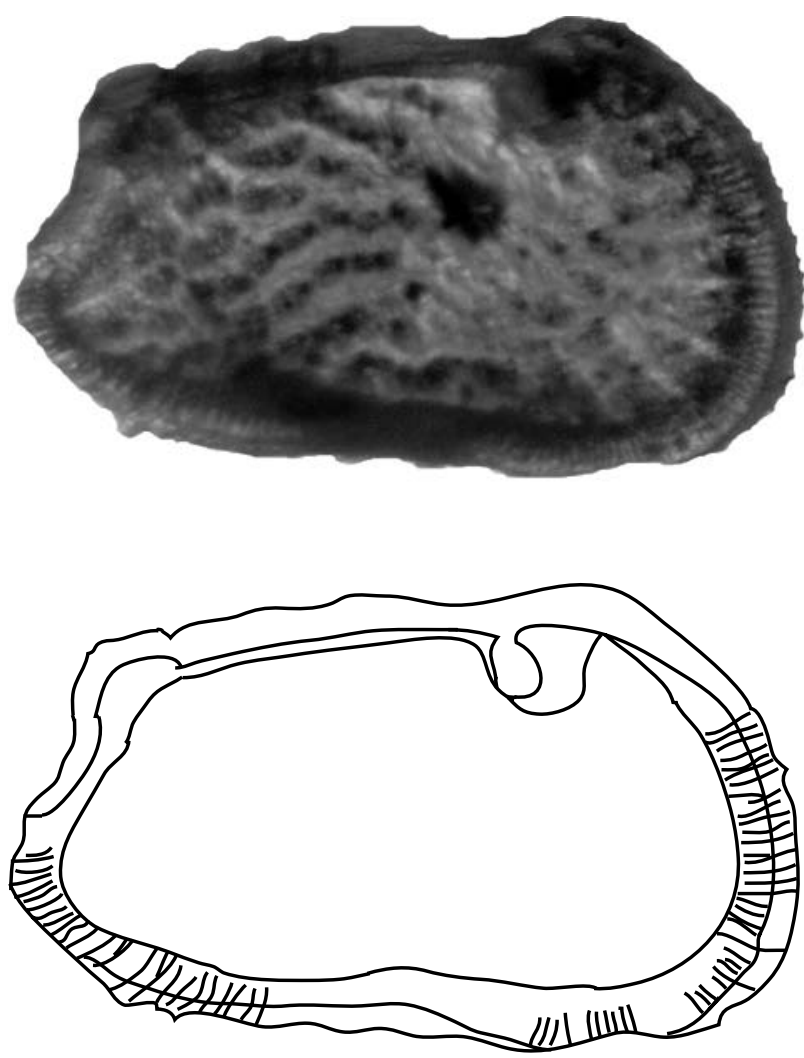

Fig. 2. View of Ambostracon irizukii sp. nov. and its trace with transmitted light. Paratype USNM 537696. Scale bar $0.1 \mathrm{~mm}$.

Material. Six adult carapaces, eleven A-1 carapaces and one A-1 left valve, including types.

Type locality and horizon. CSUN loc. 1563. See also Appendix A.

Description. Carapace robust and large in size. Lateral outline subrectanglar: anterior margin round; posterior margin curved near middle of the margin, projected at lower part forming a caudal process; dorsal margin arched; ventral margin slightly sinuate, having concavely curve near anterior one-quarter. Maximum length across caudal process; maximum height across anterodorsal corner. Surface ornamented by carinae and reticulation. Dorsal and ventral outlines lenticular: anterior and posterior ends angular; lateral margins curved. Contact line of valves straight.

A-1 carapace robust and medium. Surface ornamented with fainter muri and smaller fossae than adult carapace. Internal view of left valve, amphidont-type hinge: anterior element composed of round socket and tooth in anterior order; median element smooth; posterior element round socket. Marginal infold developed. Anterior marginal pore canals straight and at least 41. Posterior marginal pore canals straight and number at least 24 .
Dimensions. See also Table 2. USNM 537692, L $=0.94 \mathrm{~mm}$, $\mathrm{H}=0.54 \mathrm{~mm}, \mathrm{~W}=0.54 \mathrm{~mm}$; USNM $537693, \mathrm{~L}=0.67 \mathrm{~mm}$, $\mathrm{H}=0.34 \mathrm{~mm}, \mathrm{~W}=0.25 \mathrm{~mm} ; \quad$ USNM $537694, \mathrm{~L}=0.87 \mathrm{~mm}$, $\mathrm{H}=0.48 \mathrm{~mm}, \mathrm{~W}=0.47 \mathrm{~mm}$; USNM $537695, \mathrm{~L}=0.61 \mathrm{~mm}, \mathrm{H}=$ $0.35 \mathrm{~mm}, \mathrm{~W}=0.29 \mathrm{~mm}$; USNM $537696, \mathrm{~L}=0.60 \mathrm{~mm}, \mathrm{H}=0.35 \mathrm{~mm}$; UWBM no. $98693, \mathrm{~L}=0.94 \mathrm{~mm}, \mathrm{H}=0.53 \mathrm{~mm}, \mathrm{~W}=0.52 \mathrm{~mm}$; UWBM no. $98694, \mathrm{~L}=0.69 \mathrm{~mm}, \mathrm{H}=0.38 \mathrm{~mm}, \mathrm{~W}=0.20 \mathrm{~mm}$.

Occurrence. CSUN loc. 1563 and 1564.

Remarks. The subrectangular lateral outline, reticulation with a distinct ventral murus, and a non-projected subcentral tubercle are characters of Ambostracon (Hazel, 1962). In addition, the number of marginal pore-canals of the A-1 specimen confirms that this species belongs to the genus Ambostracon (Hazel, 1967; Irizuki, 1993). Ambostracon irizukii sp. nov. resembles Ambostracon sp. D of Valentine (1976) from modern sediments of the west coast of North America in the regular pattern of polygonal fossae. The new species differs from Ambostracon sp. D by having narrower muri and less reticulation on the anterodorsal area.

\section{Genus Coquimba Ohmert, 1968}

Coquimba washingtonensis sp. nov. (Pl. 2, figs 14, 15; Pl. 3, figs 1-3)

Derivation of name. Named for Washington State.

Diagnosis. A Coquimba partly ornamented with feeble reticulation formed by polygonal fossae.

Holotype. USNM 537697, adult carapace from CSUN loc. 1563.

Paratypes. USNM 537698, adult carapace from CSUN loc. 1563; USNM 537699, adult carapace from CSUN loc. 1563; USNM 537700, adult carapace from CSUN loc. 1563; UWBM no. 98695 , adult carapace from CSUN loc. 1563; UWBM no. 98696, adult carapace from CSUN loc. 1563.

Material. Fourteen adult carapaces, including types.

Type locality and horizon. CSUN loc. 1563. See also Appendix A.

Description. Carapace slightly robust and medium to large in size. Lateral outline subrectanglar: anterior margin round; posterior margin angular; dorsal margin straight; ventral margin slightly curved. Maximum length across the middle of carapace. Maximum height across anterodorsal corner. Surface ornamented with feeble reticulation and ventral carina. Reticulation formed by irregular and polygonal fossae, commonly developed in central area. Subcentral tubercle feebly present. Ventral carina running horizontally and expanded as ala. Eye tubercle distinct. Dorsomedian area slightly depressed. Anterior and posterior marginal rims developed. Marginal denticles projected from antero- and postero-ventral margins: antero-ventral marginal denticles thick; postero-ventral margins small. Dorsal and ventral margins elongated hexagonally: anterior and posterior 

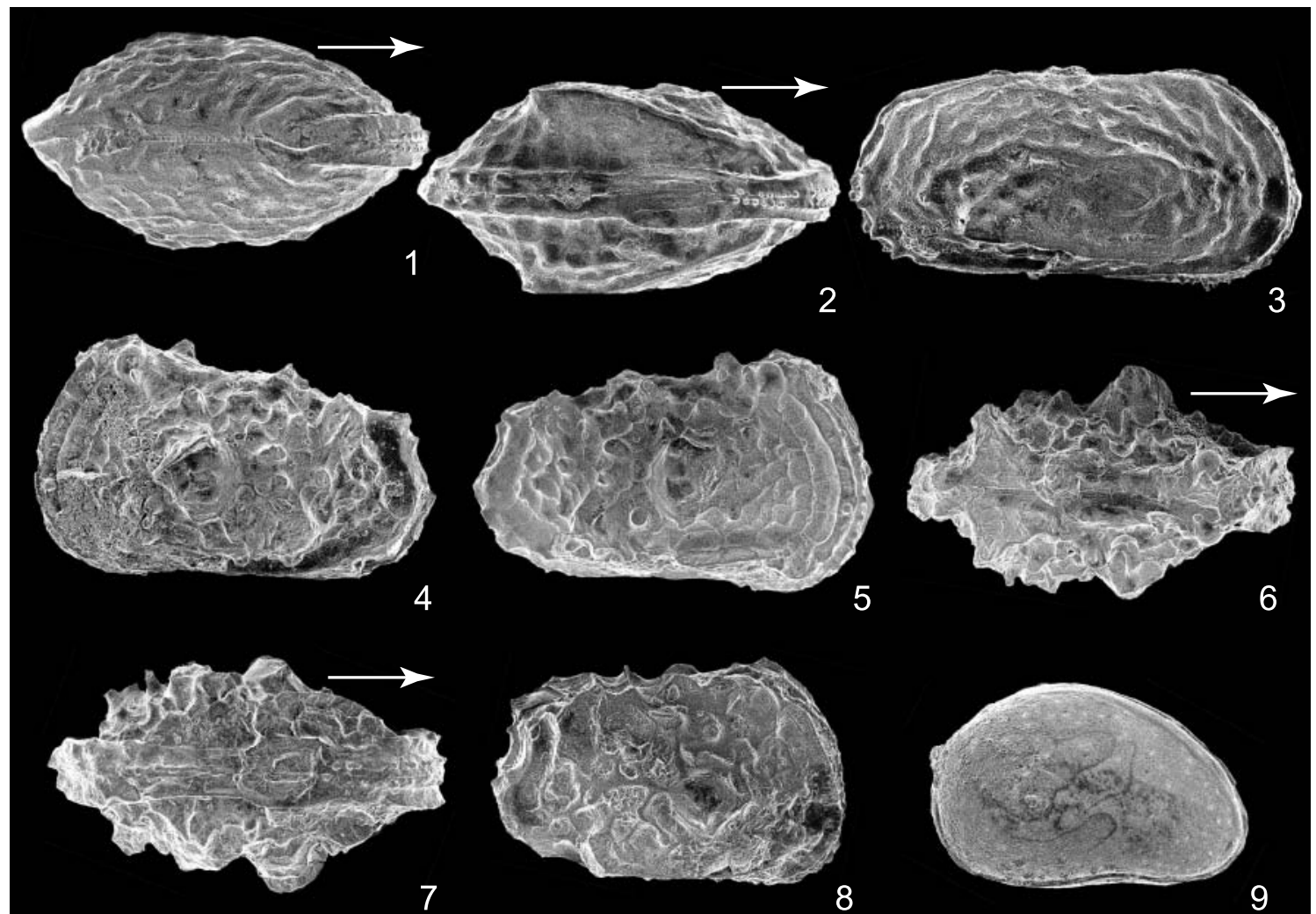

9
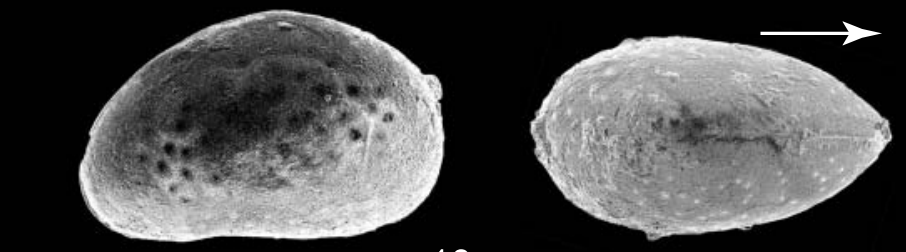

10

8
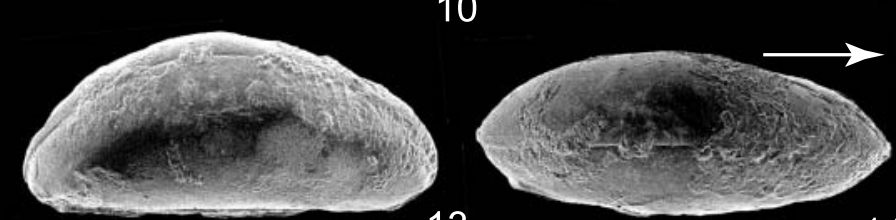

11
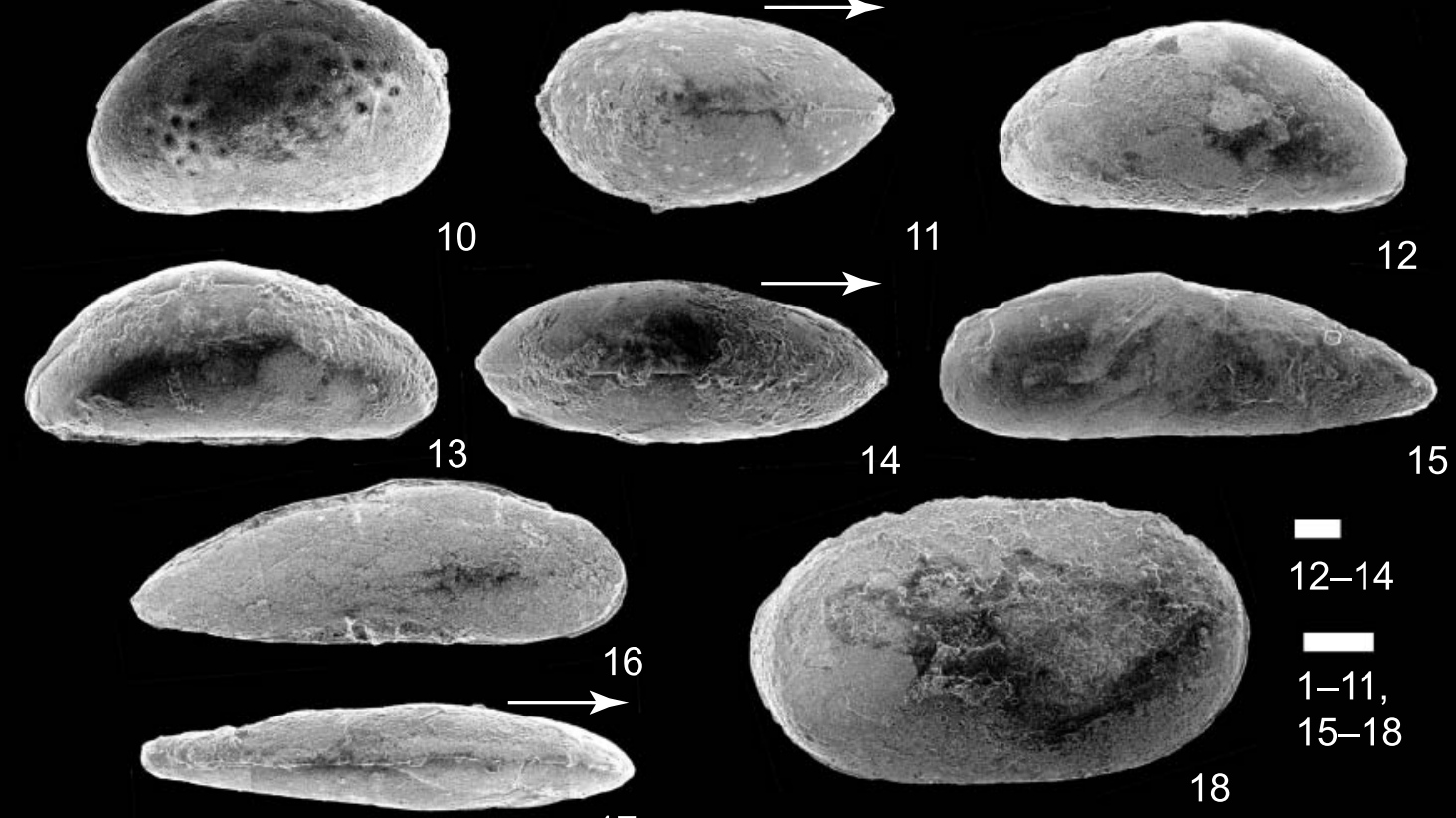

16

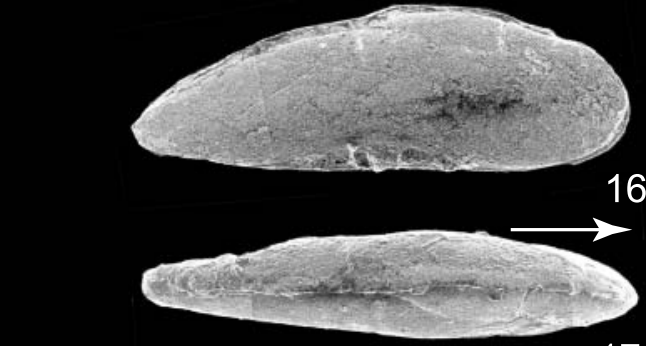

14

17

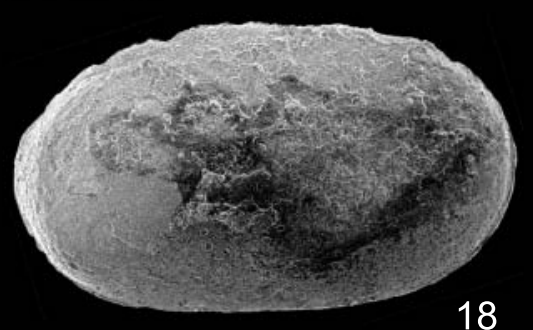

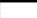

12-14

1-11,

15-18

18

Explanation of Plate 3.

figs 1-3. Coquimba washingtonensis sp. nov.: 1, dorsal view, holotype, USNM 537697, adult carapace; 2, ventral view, holotype, USNM 537697, adult carapace; 3, right lateral view, paratype, USNM 537698, adult carapace. figs 4-8. Acanthocythereis olympiana sp. nov.: 4, left lateral view, holotype, USNM 537701, adult male carapace; 5, right lateral view, holotype, USNM 537701, adult male carapace; 6, dorsal view, holotype, USNM 537701, adult male carapace; 7, ventral view, holotype, USNM 537701, adult male carapace; 8, right lateral view, paratype, USNM 537702, adult female carapace. figs 9-11. Xestoleberis sp., USNM537704, adult carapace: 9, left lateral view; 10, right lateral view; 11, dorsal view. figs 12-14. Parakrithella sp., USNM537705, adult carapace: 12, left lateral view; 13, right lateral view; 14, dorsal view. figs 15-17. Paradoxostoma sp., USNM537706, adult carapace: 15, left lateral view; 16, right lateral view; 17, dorsal view. fig. 18. Cytherella sp., USNM537707, adult left valve, external view. Scale bars $0.1 \mathrm{~mm}$. Arrows indicate anterior direction. All specimens are from CSUN loc. 1563. 
margins angular. Contact line of valves across middle of carapace, generally straight, but curved dextrally at anterodorsal corner. Alae forming flattened ventral surface.

Dimensions. See also Table 2. USNM 537697, L=0.74 mm, $\mathrm{H}=0.38 \mathrm{~mm}, \mathrm{~W}=0.37 \mathrm{~mm} ; \quad$ USNM 537698, $\mathrm{L}=0.78 \mathrm{~mm}$, $\mathrm{H}=0.35 \mathrm{~mm}, \mathrm{~W}=0.40 \mathrm{~mm} ; \quad$ USNM 537699, L=0.73 mm, $\mathrm{H}=0.35 \mathrm{~mm}, \mathrm{~W}=0.36 \mathrm{~mm}$; USNM 537700, $\mathrm{L}=0.75 \mathrm{~mm}$, $\mathrm{H}=0.38 \mathrm{~mm}, \mathrm{~W}=0.36 \mathrm{~mm}$; UWBM no. $98695, \mathrm{~L}=0.70 \mathrm{~mm}$, $\mathrm{H}=0.37 \mathrm{~mm}, \mathrm{~W}=0.37 \mathrm{~mm}$; UWBM no. $98696, \mathrm{~L}=0.70 \mathrm{~mm}$, $\mathrm{H}=0.34 \mathrm{~mm}, \mathrm{~W}=0.36 \mathrm{~mm}$.

\section{Occurrence. CSUN locs 1563 and 1564.}

Remarks. The ornament of polygonal fossae is shared with Coquimba fornicata Ohmert, 1968, C. hartmanni Ohmert, 1968, C. schencki (Le Roy, 1943) and C. hanaii Brouwers, 1993. Coquimba fornicata and C. hartmanni were originally described from Pliocene strata in Chile (Ohmert, 1968), C. schencki from the Pliocene Santa Barbara Formation of California (Le Roy, 1943). Coquimba hanaii was described from Holocene sediments off Alaska and is also found in Holocene and Pleistocene sediments around Washington and California (Brouwers, 1993). Coquimba fornicata and C. hartmanni differ from C. washingtonensis sp. nov. with their more distinct reticulation and having a surface widely covered with reticulation. Coquimba schencki is distinguished from $C$. washingtonensis sp. nov. in having distinct and coarser reticulation, and a smaller carapace. Coquimba hanaii differs from $C$. washingtonensis sp. nov. in having a larger carapace, finer reticulation and broader flattened anterior and posterior areas.

Family Trachyleberididae Sylvester-Bradley, 1948 Genus Acanthocythereis Howe, 1963

Acanthocythereis olympiana sp. nov. (P1. 3, figs 4-8)

Derivation of name. Named for the city of Olympia, Washington.

Diagnosis. A small Acanthocythereis characterized by less reticulation on anterior area and thicker spines.

Holotype. USNM 537701, adult male carapace from CSUN loc. 1563.

Paratypes. USNM 537702, adult female carapace from CSUN loc. 1563; USNM 537703, adult female carapace from CSUN loc. 1563; UWBM no. 98697, adult female carapace from CSUN loc. 1563 .

Type locality and horizon. CSUN loc. 1563. See also Appendix A.

Description. Carapace robust and medium in size. Lateral outline subtrapezoidal: anterior margin round; posterior margin tapering at middle of this margin, forming caudal process; dorsal margin slightly arched; posterior margin straight or slightly curved. Maximum length across anterodorsal corner; maximum height across middle of carapace.
Surface ornamented with reticulation and spines. Reticulation concentric, centred around subcentral tubercle, formed by mainly rectangular fossae and faint muri with conjunctive spines. Blunt and distinct spines present on posterior area and along dorsal margin. Subcentral tubercle distinct. Eye tubercle prominent.

Dorsal outline elliptical: anterior and posterior ends angular; lateral outline undulated. Maximum width across subcentral tubercle.

Dimensions. See also Table 2. USNM 537701, L=0.70 mm, $\mathrm{H}=0.41 \mathrm{~mm}, \mathrm{~W}=0.41 \mathrm{~mm} ; \quad \mathrm{USNM}$ 537702, $\mathrm{L}=0.61 \mathrm{~mm}$, $\mathrm{H}=0.39 \mathrm{~mm}, \mathrm{~W}=0.34 \mathrm{~mm}$; USNM 537703, $\mathrm{L}=0.61 \mathrm{~mm}$, $\mathrm{H}=0.39 \mathrm{~mm}, \mathrm{~W}=0.34 \mathrm{~mm}$; UWBM no. $98697, \mathrm{~L}=0.63 \mathrm{~mm}$, $\mathrm{H}=0.37 \mathrm{~mm}, \mathrm{~W}=0.34 \mathrm{~mm}$.

Remarks. A carapace surface covered with conspicuous spines and less reticulation is shared with Acanthocythereis dunelmensis (Norman, 1865) and A. japonica Irizuki \& Yamada in Irizuki et al. (2004). Acanthocythereis dunelmensis is widely distributed in the North Atlantic and North Pacific today (e.g. Athersuch et al., 1989; Tanaka et al., 2002). Acanthocythereis japonica was originally described from the Lower Miocene Toyama Formation in central Japan. Acanthocythereis olympiana sp. nov. has a smaller carapace covered with thicker spines than either $A$. dunelmensis or A. japonica.

\section{DISCUSSION}

Globerman et al. (1982) reported benthic foraminifera from the Crescent Formation in the Black Hills, suggesting water depths shallower than $50 \mathrm{~m}$ near CSUN loc. 1563. Squires \& Goedert (1994) reported a molluscan assemblage from CSUN locs 1563 and 1564 that was comprised of intertidal and shallow-subtidal taxa. Observing the lithofacies and the mode of occurrence of the intertidal taxa, Squires \& Goedert (1994) inferred that the intertidal taxa had been transported a short distance seaward with muddy debris and clasts of basalt and were then deposited in cracks between boulders of basalt. The ostracod fauna from these two localities also contains intertidal taxa and shallowsubtidal taxa. Species of Bairdoppilata, Paradoxostoma and Xestoleberis have been reported living in sea-grasses and bottom sediments in modern intertidal zones (e.g. Maddocks, 1969; Athersuch et al., 1989). On the other hand, species of Acanthocythereis, Ambostracon, Coquimba, Cytherella and Cytherelloidea live in subtidal to shelf environments (e.g. Valentine, 1976). Apparently, the mollusc and ostracod intertidal taxa were transported together for a short distance and mixed with other taxa from subtidal to shelf environments.

The assemblage from CSUN locs 1563 and 1564 does not share any species with the faunas of the Capay Formation in California described by Marianos \& Valentine (1958). Generally, changes in modern ostracode faunas correspond to depositional environments (e.g. Smith \& Horne, 2002). Unruh et al. (1995) analysed Cretaceous-Palaeogene depositional systems in California and regarded the Capay Formation to be a fill deposit of a submarine canyon. Thus, the only Early Eocene ostracodes previously reported from western North America are from strata representing deeper water than the palaeoenvironment preserved in the Crescent Formation in the Black Hills, 


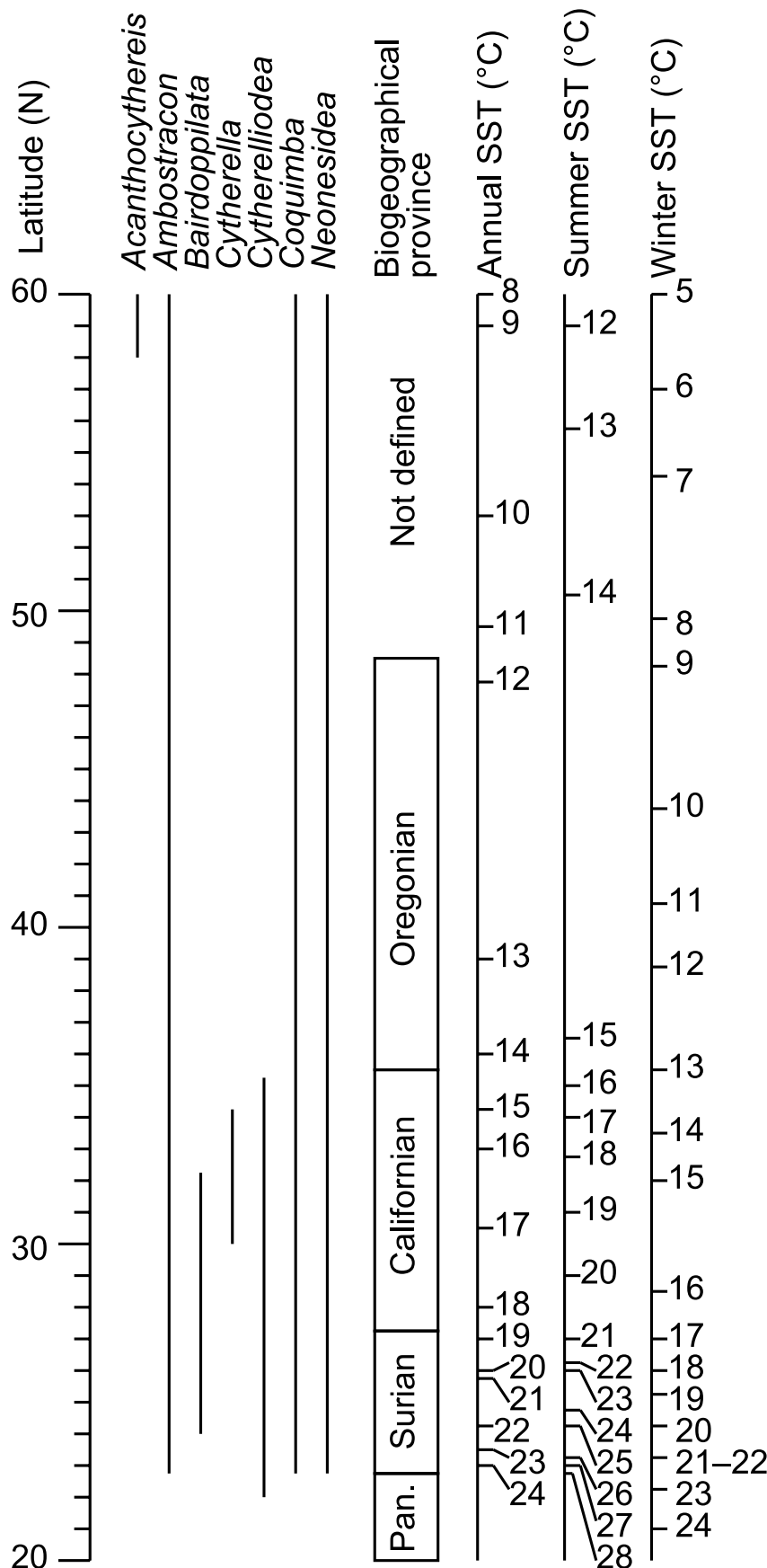

Fig. 3. Holocene geographical distribution of ostracode genera in the Pacific Northwest (Swain \& Gilby, 1974; Valentine, 1976; Brouwers, 1990, 1993). Biogeographical provinces and sea surface temperatures data are from Valentine et al. (1976) and Locamini et al. (2006), respectively. Pan., Panamanian; SST, sea surface temperature.

Washington State. The difference in faunal composition between the assemblages from California and Washington reflects the difference in depositional environments.

The Crescent Formation ostracod assemblage contains genera living in both the low and high latitudes of the Pacific Coast. Living species of Ambostracon and Coquimba are distributed between $22^{\circ} \mathrm{N}$ and $70^{\circ} \mathrm{N}$ latitudes, whereas Bairdoppilata and Cytherella are tropical-subtropical taxa that inhabit regions far south of Washington (Valentine, 1976; Brouwers, 1990, 1993; Fig. 3) and Bairdoppilata occurs abundantly in tropical and subtropical realms (Maddocks, 1969). Acanthocythereis is distributed in the high latitudes of the Pacific Coast at the present. The Early Eocene latitude of Washington was almost the same as today (Wells, 1990). The extant tropical-subtropical genera indicate that in Early Eocene time the climate in the area that now forms western Washington was much warmer than today. Acanthocythereis is rare in the Crescent Formation assemblages. The modern distribution of Acanthocythereis is controlled by cold sea-temperatures; however, the genus lived around the Gulf Coast of Mexico during the Eocene (e.g. Howe, 1963) so it appears to have adapted to cooler temperatures after Eocene time. In addition, Neomonoceratina occurs in Palaeogene strata around the Tethys Sea (e.g. McKenzie, 1983), whereas Neomonoceratina does not live along the Pacific coast today (Valentine, 1976). Consequently the Crescent Formation ostracod assemblage indicates tropical to subtropical conditions consistent with the palaeoecology of the associated warm-water molluscan fauna (Squires \& Goedert, 1994, 1996; Groves \& Squires, 1995).

A climate model also shows that Early Eocene sea surface temperature (SST) around the area that is now Washington State was higher than it is today. Huber \& Caballero (2003) performed a general circulation model of Early Eocene climates and predicted that annual SST was $13-17^{\circ} \mathrm{C}$ in the eastern North Pacific Ocean at $40^{\circ}-50^{\circ} \mathrm{N}$ latitudes. In contrast, today the annual SST around Washington State is $11-13^{\circ} \mathrm{C}$ (Locarnini et al., 2006; Fig. 3). The Early Eocene Climatic optimum correlates to the 'Capay' molluscan stage, and the Crescent Formation ostracod fauna contains warm-water taxa adapted to conditions that prevailed during the climatic optimum.

\section{ACKNOWLEGEMENTS}

Some samples were processed in the laboratories of Dr Hiroshi Kurita, Niigata University and of Prof. Takahiro Kamiya, Kanazawa University and the authors are deeply grateful to them. The authors are also grateful to Drs Thomas Cronin, John Gregory and an anonymous reviewer for their reviews and valuable comments. Thanks to Mr Mark Florence and Ronald Eng for their help to register the specimens with the National Museum of Natural History, Smithsonian Institution and the Burke Museum of Natural History and Culture, University of Washington, Seattle.

\section{Manuscript received 13 October 2008 Manuscript accepted 5 October 2009}

\section{APPENDIX A: LOCALITIES CITED}

- CSUN loc. 1563 (=LACMIP loc. $16655=$ UWBM loc. B6169 'Larch Mountain'). At elevation of $680 \mathrm{~m}$, exposed in a road cut on the northeast side of logging road, Approx. coordinates are Lat. $47^{\circ} 59^{\prime} 03^{\prime \prime} \mathrm{N}$., Long. $123^{\circ} 8^{\prime} 12^{\prime \prime} \mathrm{W}, 300 \mathrm{~m}$ north and $50 \mathrm{~m}$ east of southwest of corner section $1, \mathrm{~T} 17 \mathrm{~N}, \mathrm{R} 4 \mathrm{~W}$, and $500 \mathrm{~m} \mathrm{~S} 32^{\circ} \mathrm{E}$ of Larch Mountain, Capitol Peak US Geological Survey quadrangle, 7.5-minute, 1986, Thurston County, Washington. 
- CSUN loc. 1564 (=LACMIP loc. 16848). At elevation of $530 \mathrm{~m}$, on the north side of logging road, $800 \mathrm{~m}$ north and $50 \mathrm{~m}$ west of southwest corner of section 25, T18N, R4W and $950 \mathrm{~m} \mathrm{~N} 25^{\circ} \mathrm{W}$ of Rock Candy Mountain, Summit Lake US Geological Survey quadrangle, 7.5-minute, 1981, Thurston County, Washington.

\section{REFERENCES}

Alexander, C.I. 1929. Ostracoda of the Cretaceous of north Texas. University of Texas Bulletin, Bureau of Economic Geology, 2907: 7-114.

Almgren, A.A., Filewicz, M.V. \& Heitman, H.L. 1988. Lower Tertiary foraminiferal and calcareous nannofossil zonation of California: An overview and recommendation. In: Filewicz, M.V. \& Squires, R.L. (Eds), Paleogene stratigraphy, West Coast of North America. Pacific Section, SEPM, 58: 83-106.

Armentrout, J.M. \& Worsley, T.R. 1980. Tertiary calcareous nannofossils from southwest Washington. Newsletters in Stratigraphy, 9: 58-71.

Arnold, R. 1906. A geological reconnaissance of the coast of the Olympic Peninsula, Washington. Geological Society of America Bulletin, 17: 451-468.

Athersuch, J., Horne, D.J. \& Whittaker, J.E. 1989. Marine and Brackish Water Ostracods. The Linnaean Society of London and the Estuarine and Brackish-Water Sciences Association, UK, London, 343 pp.

Babcock, R.S., Suczek, C.A. \& Engebretson, D.C. 1994. The Crescent 'Terrane', Olympic Peninsula and southern Vancouver Island. Washington Division of Geology and Earth Resources Bulletin, 80: 141-157.

Baird, W. 1850. The Natural History of the British Entomostraca. Ray Society, London, 364 pp.

Berggren, W.A. \& Miller, K.G. 1988. Paleogene tropical planktonic foraminiferal biostratigraphy and magnetobiochronology. Micropaleontology, 34: 362-380.

Berggren, W.A., Kent, D.V., Swisher, C.C. \& Aubry, M.-P. 1995. A revised Cenozoic geochronology and chronostratigraphy. In: Berggren, W.A., Kent, D.V., Aubry, M.-P. \& Hardenbol, J. (Eds), Geochronology, Time Scales and Global Stratigraphic Correlation, SEPM Special Publication, 54: 129-212.

Brouwers, E.M. 1990. Systematic paleontology of Quaternary ostracode assemblages from the Gulf of Alaska, part 1: Families Cytherellidae, Bairdiidae, Cytheridae, Leptocytheridae, Limnocytheridae, Eucytheridae, Krithidae, Cushmanideidae. US Geological Survey Professional Paper, 1510: 1-43.

Brouwers, E.M. 1993. Systematic paleontology of Quaternary ostracode assemblages from the Gulf of Alaska, part 2: Families Trachyleberididae, Hemicytheridae, Loxoconchidae, Paracytherideidae. US Geological Survey Professional Paper, 1531: 1-47.

Carreño, A.L. \& Cronin, T.M. 1993. Middle Eocene Ostracoda from Baja California Sur, Mexico. Journal of Micropalaeontology, 12: $141-153$

Coryell, H.N., Sample, C.H. \& Jennings, P.H. 1935. Bairdoppilata, a new genus of Ostracoda, with two new species. American Museum Novitates, 777: 1-5.

Cronin, T.M. 1991. Late Neogene marine Ostracoda from Tjörnes, Iceland. Journal of Paleontology, 65: 767-794.

Givens, C.R. 1974. Eocene molluscan biostratigraphy of the Pine Mountain area, Ventura County, California. University of California Publication in Geological Sciences, 109: 1-107.

Globerman, B.R., Beck, M.E. Jr. \& Duncan, R.A. 1982. Paleomagnetism and tectonic significance of Eocene basalts from Black Hills, Washington Coast Range. Bulletin of the American Geological Society, 93: $1151-1159$.

Groves, L.T. \& Squires, R.L. 1995. First report of the genus Proadusta Sacco, 1894 (Gastropoda: Cypraeidae) from the Western Hemisphere, with a description of a new species from the Eocene of Washington. The Nautilus, 109: 113-116.

Hartmann, G. \& Puri, H.S. 1974. Summary of neontological and paleontological classification of Ostracoda. Mitteilungen aus dem Hamburgischen Zoologischen Museum und Institut, 70: 7-73.
Hazel, J.E. 1962. Two new hemicytherid ostracods from the Lower Pleistocene of California. Journal of Paleontology, 36: 822-826.

Hazel, J.E. 1967. Classification and distribution of the Recent Hemicytheridae and Trachyleberididae (Ostracoda) off northeastern North America. US Geological Survey Professional Paper, 564: 1-49.

Holden, J.C. 1964. Upper Cretaceous ostracods from California. Palaeontology, 7: 393-429.

Horne, D.J., Cohen, A. \& Martens, K. 2002. Taxonomy, morphology and biology of Quaternary and living Ostracoda. In: Holmes, J.A. \& Chivas, A.R. (Eds), The Ostracoda: Applications in Quaternary Research. American Geophysical Union, Washington D.C. Geophysical Monograph, 131: 5-36.

Howe, R.C. 1963. Type Saline Bayou Ostracoda of Louisiana. Geological Bulletin, State of Louisiana Department of Conservation, Louisiana Geological Survey, 40: 1-62.

Huber, M.T. \& Caballero, R. 2003. Eocene El Niño: Evidence for robust tropical dynamics in the 'Hothouse'. Science, 299: 877-881.

Irizuki, T. 1993. Morphology and taxonomy of some Japanese hemicytherin Ostracoda - with particular reference to ontogenetic changes of marginal pores. Transactions and Proceedings of the Palaeontological Society of Japan, New Series, 170: 186-211.

Irizuki, T., Yamada, K., Maruyama, T. \& Ito, H. 2004. Paleoecology and taxonomy of Early Miocene Ostracoda and paleoenvironments of the eastern Setouchi Province, central Japan. Micropaleontology, 50: 105-147.

Kingma, J.T. 1948. Contribution to the knowledge of the young Caenozoic Ostracoda from the Malayan Region. Kemink Printers, Utrechet, $108 \mathrm{pp}$.

Lear, C.H. 2007. Mg/Ca palaeothermometry: a new window into Cenozoic climate change. In: Williams, M. et al. (Eds), Deep-Time Perspectives on Climate Change: Marrying the Signal from Computer Models and Biological Proxies. The Micropalaeontological Society Special Publications, The Geological Society, London, 313-322.

Lear, C.H., Elderfield, H. \& Wilson, P.A. 2000. Cenozoic deep-sea temperatures and global ice volume from $\mathrm{Mg} / \mathrm{Ca}$ in benthic foraminiferal calcite. Science, 287: 269-272.

Le Roy, L.W. 1943. Pleistocene and Pliocene Ostracoda of the coastal region of southern California. Journal of Paleontology, 17: 354-373.

Liu, Z. 1989. Ostracoda. In: Research Party of Marine Geology, Ministry of Geology and Mineral Resources, Institute of Geology, Chinese Academy of Geological Science (Ed.), Cenozoic Paleobiota of the Continental Shelf of the East China Sea (Donghai), 136-163. Geological Publishing House, Beijing [in Chinese with English abstract].

Locarnini, R.A., Mishonov, A.V., Antonov, J.I., Boyer, T.P. \& Garcia, H.E. 2006. Volume 1: Temperature. In: Levitus, S. (Ed.), World Ocean Atlas 2005. NOAA Atlas NESDIS 61, US Government Printing Office, Washington, D.C., 182 pp.

Luterbacher, H.P., Ali, J.R., Brinkhuis, H. et al. 2004. The Paleogene Period. In: Gradstein, F.M., Ogg, J.G. \& Smith, A. (Eds), A Geologic Time Scale 2004, 384 408. Cambridge University Press, Cambridge.

Maddocks, R. 1969. Revision of Recent Bairdiidae (Ostracoda). US National Museum, Bulletin, 295: 1-126.

Mallory, V.S. 1959. Lower Tertiary biostratigraphy of the California Coast Range. American Association of Petroleum Geologists, Tulsa, OK, 416 pp.

Marianos, A.W. \& Valentine, J.W. 1958. Eocene ostracode fauna from Marysville Buttes, California. Micropaleontology, 4: 363-372.

Martini, E. 1971. Standard Tertiary and Quaternary calcareous nannoplankton zonation. In: Farinaccia, A. (Ed.), Proceedings of the 2nd International Conference on Planktonic microfossils, Rome 1970, Tecnoscienza, Rome, 739-985.

McKenzie, K.G. 1983. Shallow marine and freshwater Tethyan Ostracoda: their usefulness for petroleum exploration. In: Maddocks, R.F. (Ed.), Application of Ostracoda, 35-50. University of Houston Geosciences, Houston.

Norman, A.M. 1865. Deep-sea dredging on the coasts of Northumberland and Durham, in 1864. British Association for the Advancement of Science Report, 34th Meeting, 189-193.

Ohmert, V.W. 1968. Die Coquimbinae, eine neue Unterfamilie der Hemicytheridae (Ostracoda) aus dem Pliozän von Chile. Mitteilungen 
der Bayerischen Staatssammlung für Paläontologie und Historische Geologie, 8: 127-165.

Okada, H. \& Bukry, D. 1980. Supplementary modification and introduction of code numbers to the low-latitude coccolith biostratigraphic zonation (Bukry, 1973; 1975). Marine Micropaleontology, 5: 321-325.

Puri, H.S. 1953. The ostracod genus Hemicythere and its allies. Washington Academy of Sciences Journal, 43: 169-179.

Sars, G.O. 1866. Oversigt af Norges marine Ostracoder: Forhandlinger I. Videnskabs-Selskabet I Christiania, 8: 1-130.

Sars, G.O. 1888. Nye bidrag til kundskaben om middlehavets invertebratfauna, 4. Ostracoda Mediterranea. Archiv for Mathematik og Naturvidenskab, 12: 173-324.

Smith, A.J. \& Horne, D.J. 2002. Ecology of marine, marginal marine and nonmarine ostracodes. In: Holmes, J.A. \& Chivas, A.R. (Eds), The Ostracoda: Applications in Quaternary Research. American Geophysical Union, Washington D.C. Geophysical Monograph, 131: 37-64.

Squires, R.L. 1988. Geologic age refinements of west coast Eocene marine mollusks. In: Filewicz, M.V. \& Squires, R.L. (Eds), Paleogene stratigraphy, West Coast of North America, Pacific Section SEPM, 107-112.

Squires, R.L. \& Goedert, J.L. 1994. New species of early Eocene small to minute mollusks from the Crescent Formation, Black Hills, southwestern Washington. The Veliger, 37: 253-266.

Squires, R.L. \& Goedert, J.L. 1996. New species of small to minute gastropods of Early Eocene age from the Crescent Formation, Black Hills, Southwest Washington. The Veliger, 39: 226-240.

Swain, F.M. \& Gilby, J.M. 1974. Marine Holocene Ostracoda from the
Pacific coast of North and Central America. Micropaleontology, 20: 257-352.

Sylvester-Bradley, P.C. 1948. The ostracod genus Cythereis. Journal of Paleotology, 22: 792-797.

Tanaka, G., Seto, K., Mukuda, T. \& Nakano, Y. 2002. Middle Miocene ostracods from the Fujina Formation, Shimane Prefecture, Southwest Japan and their paleoenvironmental significance. Paleontological Research, 6: 1-22.

Unruh, J.A., Loewen, B.A. \& Moores, E.M. 1995. Progressive arcward contraction of a Mesozoic-Tertiary fore-arc basin, southwestern Sacramento Valley, California. The Geological Society of America Bulletin, 107: 38-57.

Valentine, P.C. 1976. Zoogeography of Holocene Ostracoda off Western North America and paleoclimatic implications. USGS Professional Paper, 916: 1-47.

van Veen, J.E. 1936. Die Cytheridae der Maastrichter Tuffkreide und des Kunrader Korallenkalkes von Sud-Limburg. Natuurhistorisch Maandblad, 25: 21-113.

Wells, R.E. 1990. Paleomagnetic rotations and the Cenozoic tectonics of the Cascade Arc, Washington, Oregon, and California. Journal of Geophysical Research, 95B: 19409-19417.

Yang, H., Chen, D. \& Wang, J. 1990. Eocene Ostracoda from the southwestern Continental Shelf of the East China Sea. Acta Micropalaeontologica Sinica, 7: 367-388 [in Chinese with English abstract). Zachos, J., Pagani, M., Sloan, L., Thomas, E. \& Billups, K. 2001. Trends, rhythms, and aberrations in global climate 65 Ma to Present. Science, 292: 686-693. 